\title{
Successful Treatment of Hepatosplenic T Cell Lymphoma in an Adolescent with Turner Syndrome Using Ifosfamide, Carboplatin, and Etoposide Followed by Allogeneic Hematopoietic Stem Cell Transplant
}

\author{
Colette Lauhan ${ }^{1}$, Deborah Schiff ${ }^{2}$, and Nicholas Gloude ${ }^{3}$ \\ ${ }^{1}$ University of California San Diego \\ ${ }^{2}$ Rady Children's Hospital, San Diego \\ ${ }^{3}$ Rady Children's Hospital San Diego
}

May 7, 2020

Abbreviations key:

\begin{tabular}{ll}
\hline Abbreviation & Full term/phrase \\
\hline HSTCL & hepatosplenic T cell lymphoma \\
CT & computed tomography \\
PET/CT & positron emission tomography/computed tomography \\
ICE & ifosfamide, carboplatin, and etoposide \\
MRD & minimal residual disease \\
HSCT & hematopoietic stem cell transplant \\
NCCN & National Comprehensive Cancer Network \\
IVAC & ifosfamide, cytarabine, etoposide \\
CHOP & cyclophosphamide, doxorubicin hydrochloride, vincristine sulfate, and prednisone \\
\hline
\end{tabular}

\section{MAIN TEXT}

To the editor: Hepatosplenic T Cell Lymphoma (HSTCL) is a rare malignancy that occurs most often in young adult males, and is associated with immunosuppressive medications ${ }^{1-3}$. Less than $10 \%$ of patients with HSTCL survive five years after diagnosis ${ }^{4}$, and there is no consensus for treatment, particularly for pediatric patients ${ }^{2}$.

A 14 year old female with Turner Syndrome and Crohn's disease previously treated with mercaptopurine for seven years presented following two weeks of facial and truncal rash (Figure 1), fatigue, night sweats, and hepatosplenomegaly. Mercaptopurine was discontinued 19 months prior to presentation. Laboratory evaluation revealed blast-like cells, white blood cell count $17500 / \mathrm{mm}^{3}$, hemoglobin $10.7 \mathrm{~g} / \mathrm{dL}$, platelet count $119000 / \mathrm{mm}^{3}$, and lactate dehydrogenase $5,885 \mathrm{u} / \mathrm{L}$. Bone marrow aspirate contained $31 \%$ malignant cellspositive for CD3, CD7, and CD56, and negative for alpha/beta T cell receptor - with isochromosome 7q and 
8q gain. Computed tomography (CT) and positron emission tomography/computed tomography (PET/CT) confirmed hepatosplenomegaly and hypermetabolic splenic lesions (Figure 2). The diagnostic workup was consistent with HSTCL.

She received induction therapy with ifosfamide, carboplatin, and etoposide (ICE) ${ }^{5}$. After one cycle, rash resolved and splenomegaly markedly improved. She achieved a complete response with negative PET/CT scan and $0.009 \%$ minimal residual disease (MRD) after four cycles. She underwent allogeneic hematopoietic stem cell transplant (HSCT) from a matched unrelated donor following conditioning with total body irradiation, thiotepa, and cyclophosphamide. Bone marrow aspirate obtained 30 days after HSCT had rare suspicious cells, but MRD was $<0.001 \%$. One year after HSCT, bone marrow evaluation was MRD negative and $\mathrm{PET} / \mathrm{CT}$ was negative for disease.

Patients with exposure to thiopurines and other immunomodulators have increased risk of HSTCL ${ }^{1-3,6,7}$. However, this is the first reported case of HSTCL in a patient with Turner Syndrome. While patients with Turner Syndrome have increased risk of solid tumors, increased hematologic malignancy is not reported in this group ${ }^{8,9}$. A systematic review of patients with HSTCL following immunosuppressive therapy for inflammatory bowel disease found that this cohort of patients were $>90 \%$ male $^{6}$, an interesting finding considering this female patient had only one X chromosome. Also notable is the development of malignancy after more than a year from discontinuation of immunomodulators, indicating that risk may be sustained over months to years after exposure.

Outcomes among HSTCL case series are dismal ${ }^{7,10,11}$, and treatment regimens vary widely. National Comprehensive Cancer Network (NCCN) guidelines revised January 2020 suggest ICE followed by allogeneic $\mathrm{HSCT}^{1}$. Ifosfamide, cytarabine, etoposide (IVAC) has been used with some success ${ }^{4}$; while cyclophosphamide, doxorubicin hydrochloride, vincristine sulfate, and prednisone (CHOP) has been largely accepted as inadequate ${ }^{1,10}$. While NCCN guidelines recommend allogeneic HSCT, American Society of Blood and Marrow Transplantation recommends autologous HSCT for older adults and those who have achieved complete response prior to transplant ${ }^{12}$.

In summary, we successfully treated a 14 year old with Turner syndrome and HSTCL with ICE and allogeneic HSCT. She remains in remission 16 months from diagnosis and 12 months following HSCT. Some patients with this challenging disease can achieve sustained remission with aggressive induction chemotherapy followed by consolidation with allogeneic HSCT.

\section{REFERENCES :}

1. Horwitz SM, Ansell S, Ai WZ, et al. T-Cell Lymphomas .; 2020.

2. Sandlund JT, Perkins SL. Uncommon non-Hodgkin lymphomas of childhood: Pathological diagnosis, clinical features and treatment approaches.Br J Haematol . 2015;169(5):631-646. doi:10.1111/bjh.13359

3. Jaffe ES, Nicolae A, Pittaluga S. Peripheral T-cell and NK-cell lymphomas in the WHO classification: pearls and pitfalls. Mod Pathol . 2013;26:S71-S87. doi:10.1038/modpathol.2012.181.Peripheral

4. D'Amore F, Gaulard P, Trümper L, et al. Peripheral T-cell lymphomas: ESMO Clinical Practice Guidelines for diagnosis, treatment and follow-up. Ann Oncol . 2015;26(Supplement 5):v108-v115. doi:10.1093/annonc/mdv201

5. Cairo MS. Non-Hodgkin Lymphoma. In: Lanzkowsky P, ed. Manual of Pediatric Hematology and Oncology . Fifth ed. London, UK; 2011:624-646. doi:10.1016/B978-0-12-375154-6.00020-3

6. Kotlyar DS, Osterman MT, Diamond RH, et al. A Systematic Review of Factors That Contribute to Hepatosplenic T-Cell Lymphoma in Patients With Inflammatory Bowel Disease. Clin Gastroenterol Hepatol . 2011;9(1):36-41. doi:10.1016/j.cgh.2010.09.016

7. Belhadj K, Farcet J, Bastard C, Deconinck E, Labouyrie E, Lederlin P. Hepatosplenic gamma-delta T-cell lymphoma is a rare clinicopathologic entity with poor outcome : report on a series of 21 patients.Blood $J$. 
2013;102(13):4261-4249. doi:10.1182/blood-2003-05-1675.Supported

8. Ji J, Zoller B, Sundquist J, Sundquist K. Risk of solid tumors and hematological malignancy in persons with Turner and Klinefelter syndromes: A national cohort study. Int J Cancer. 2016;139(4):754-758. doi:10.1002/ijc.30126

9. Hasle H, Olsen JH, Nielsen J, Hansen J, Friedrich U, Tommerup N. Occurrence of cancer in women with Turner syndrome. Br J Cancer . 1996;73(9):1156-1159. doi:10.1038/bjc.1996.222

10. Kontny U, Oschlies I, Woessmann W, et al. Non-anaplastic peripheral T-cell lymphoma in children and adolescents - a retrospective analysis of the NHL-BFM study group. Br J Haematol . 2015;168(6):835-844. doi:10.1111/bjh.13216

11. Weidmann E. Hepatosplenic T cell lymphoma. A review on 45 cases since the first report describing the disease as a distinct lymphoma entity in 1990. Leukemia . 2000;14(6):991-997. doi:10.1038/sj.leu.2401784

12. Kharfan-Dabaja MA, Kumar A, Ayala E, et al. Clinical Practice Recommendations on Indication and Timing of Hematopoietic Cell Transplantation in Mature T Cell and NK/T Cell Lymphomas: An International Collaborative Effort on Behalf of the Guidelines Committee of the American Society for Blood and Ma. Biol Blood Marrow Transplant . 2017;23(11):1826-1838. doi:10.1016/j.bbmt.2017.07.027

Figure legend:

Figure 1: Non-blanching violaceous rash at initial presentation. Rash was convalescent on the lower twothirds of the face (a), and macular over the trunk (b). Rash resolved completely after the first cycle of induction chemotherapy. Her family provided consent to publish these images. Photograph credit: Suhas Radhakrishna.

Figure 2: Diagnostic workup included CT of the neck, chest, abdomen, and pelvis and PET/CT. CT scan, identified the liver $17 \mathrm{~cm}$ in superior to inferior dimension without focal lesion and spleen $24 \mathrm{~cm}$ in superior to inferior dimension. Spleen was diffusely heterogeneous in attenuation with several large geographic areas of hypoattenuation. No additional areas of lymphadenopathy were identified (a). PET/CT was significant for massive splenomegaly with portions of the spleen intensely hypermetabolic with Deauville Score 5 (b).

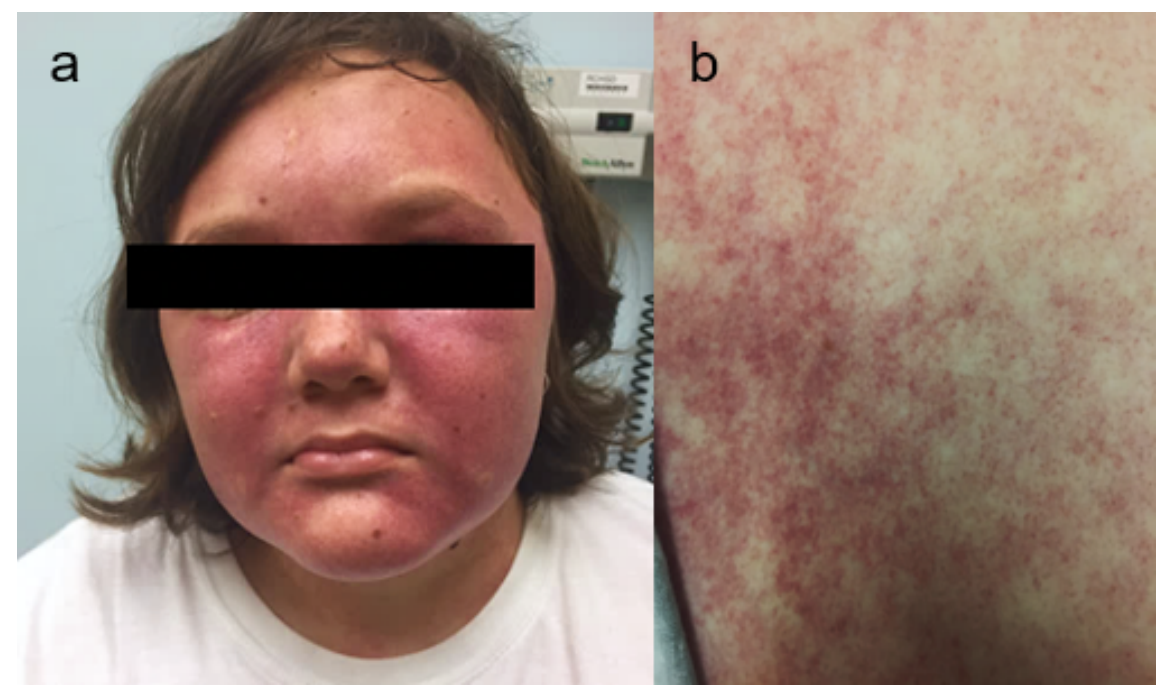




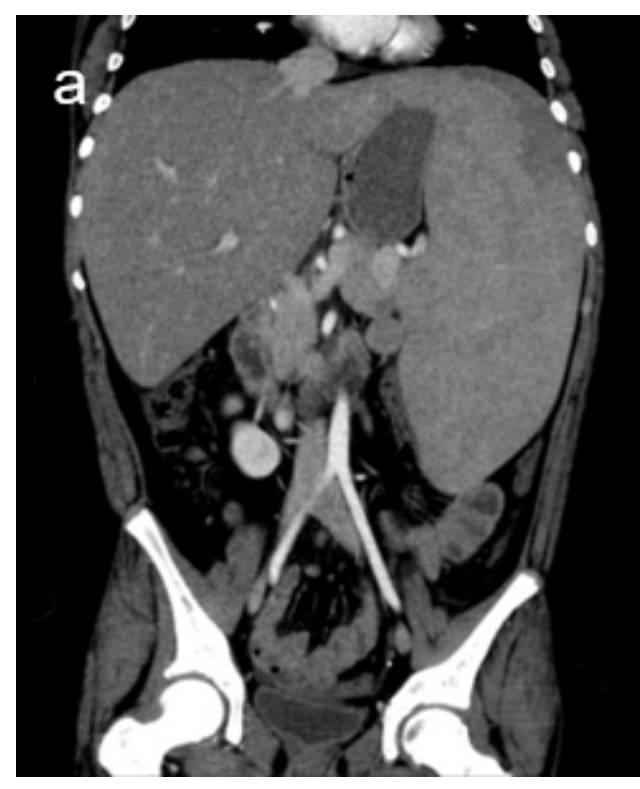

b

$8 \quad-\frac{1}{3}$

$\checkmark$ 